\title{
THE EFFECT OF THE MID-LATITUDE TROUGH ON THE DIRECTION OF ARRIVAL AND TIME-OF-FLIGHT OF HF RADIO SIGNALS
}

\author{
A.J. Stocker, E.M. Warrington and N.Y. Zaalov \\ Department of Engineering, University of Leicester, U.K.
}

\section{INTRODUCTION}

The trough is a region of depleted electron density in the night-time F-region ionosphere in which the critical frequencies drop by a factor of at least 2 (i.e. the electron density peak value drops by a factor of at least 4) and the altitude of the electron density peak rises by $100 \mathrm{~km}$ or more [1]. During the winter and equinoctial months the trough takes the form of a band a few degrees wide in latitude on the equatorward edge of the auroral oval, stretching in local time from dusk to dawn. In summer, the trough is much less pronounced and is confined to the hours around midnight. The location of the trough also depends on geomagnetic activity, the trough region moving equatorwards and the evening sector tending to move to earlier local times as the activity increases. The trough has been modelled on a statistical basis by various researchers (see, for example, [2], [3] and [4]). Recent developments in ionospheric tomography [5, 6] and observations made with EISCAT [7] have provided further experimental observations of the trough position and structure.

The presence of the mid-latitude trough has a significant impact on many radio systems, particularly those operating within the HF band where the signals are reflected from the ionosphere but also systems operating at higher frequencies where the signals traverse the ionosphere. For HF systems, the electron density depletion within the trough region reduces the maximum frequency which can be reflected by the ionosphere along the great circle path (GCP). Additionally, the associated tilts in the electron density distribution frequently result in propagation well displaced from the great circle path by up to around $100^{\circ}$ or more. These deviations impact not only on radiolocation systems for which estimates of a transmitter location are obtained by triangulation from a number of receiving sites assuming great circle propagation, but also on any radiocommunications system in which directional antennas are employed. For trans-ionospheric signals, the reduction in total electron content (TEC) over the trough region affects the time of flight of the signals which, for example, leads to timing and positional errors in satellite based navigational systems such as GPS and the proposed European Galileo system.

Observations of the effect of the trough on the azimuth-of-arrival of HF radio signals at several frequencies propagating in the sub-auroral region have been reported by Rogers et al [8]. These authors investigated the effects on two paths, the first between Halifax, Canada and Cheltenham, UK $\left(4490 \mathrm{~km}\right.$, bearing $\left.286^{\circ}\right)$ and the second between Halifax and Leitrim (near Ottawa), Canada $\left(910 \mathrm{~km}\right.$, bearing $\left.90^{\circ}\right)$. Some of the observations on the longer path are reproduced in this paper. For the longer path this study showed that the occurrence and nature of propagation well displaced from the great circle path is well correlated with the geomagnetic activity index $\mathrm{A}_{\mathrm{p}}$. During the winter and equinoctial months (October to March) the effect of the magnetic disturbances was particularly noticeable on the lowest frequency $(5.097 \mathrm{MHz})$ signal, where during the more disturbed days (i.e. when $A_{p} \geq 15$ ) a characteristic high-to-low bearing angle swing of about $50^{\circ}$ was evident. Furthermore, greatly increased Doppler spread occurs on those days when large offsets from the great circle path are present. In the summer months however, there is a disproportionately low occurrence of the characteristic bearing changes which is consistent with the well documented absence of the trough during this period. A systematic dependence on $A_{p}$ was also observed on higher frequency transmissions (10.945 MHz and $15.920 \mathrm{MHz}$ ). During periods of low geomagnetic activity, propagation is often at bearings equatorwards of the great circle path (GCP) and the time of occurrence of the change to negative bearings is correlated with $A_{p}$. The trend to earlier occurrence for increasing $A_{p}$ is very clear and is consistent with the expected extension of the sub-auroral trough into earlier local times in the evening sector during enhanced geomagnetic activity. These long-path observations have been simulated using ray tracing techniques, the results of which are presented in this paper and can be explained in terms of seascatter from regions far to the south of the trough. It is also interesting to note that following the onset of a geomagnetic storm, propagation during the night is often observed to the north of the GCP.

The much shorter path from Halifax to Leitrim is at most times sub-auroral and strong effects due to the mid-latitude trough are expected. At the lowest frequency $(5.097 \mathrm{MHz})$, very large bearing deviations of up 
to $\pm 100^{\circ}$ starting at times two or three hours after midnight UT are a common feature. The deviations can be either positive, negative or include both directions even for geomagnetically quiet days. During disturbed times, the large bearing deviations tend to start a few hours earlier with the time of occurrence strongly related to $\mathrm{A}_{\mathrm{p}}$. At higher frequencies (e.g. $10.945 \mathrm{MHz}$ ) the large bearing swings are much less common since the signal is usually lost at $0000 \mathrm{UT}$ due to the low MUF and is not reacquired until about $1200 \mathrm{UT}$.

In this paper, the results of the ray tracing simulation of the deviations observed over the $4490 \mathrm{~km}$ path from Canada to the U.K. are presented together with new observations from a new experimental system deployed in Uppsala, Sweden and Leicester, U.K, (a $1400 \mathrm{~km}$ propagation path). This new system is capable of measuring more properties of the received signal than that previously employed, including the direction of arrival in both azimuth and elevation, time-of-flight (and hence delay spread), Doppler shift (and hence Doppler spread) and amplitude.

\section{RAYTRACING SIMULATION}

Observations [8] of the bearing of the $10.945 \mathrm{MHz}$ signal transmitted from Halifax, Nova Scotia and measured at Cheltenham, U.K. [8] during the period from 1200 UT, 24 March 1994 to 1200 UT, 25 March 1994 at are presented in Figure 1. Prior to about 0000 UT, propagation is close to the great circle direction $\left(286^{\circ}\right)$. However, after 0000 UT the bearing begins to turn southwards reaching a peak deviation from the great circle path of around $40-50^{\circ}$ by about 0600 UT. After this time, the southward deviation in bearing reduced, returning to the GCP by 9 UT. The geomagnetic activity during this interval was relatively low (daily $\mathrm{A}_{\mathrm{p}}$ was 18 and 17 for the 24 March and $25 \mathrm{March}$, respectively). Note that similar directional behaviour was also observed on a number of days immediately before and after this particular interval for which the geomagnetic activity was comparable.

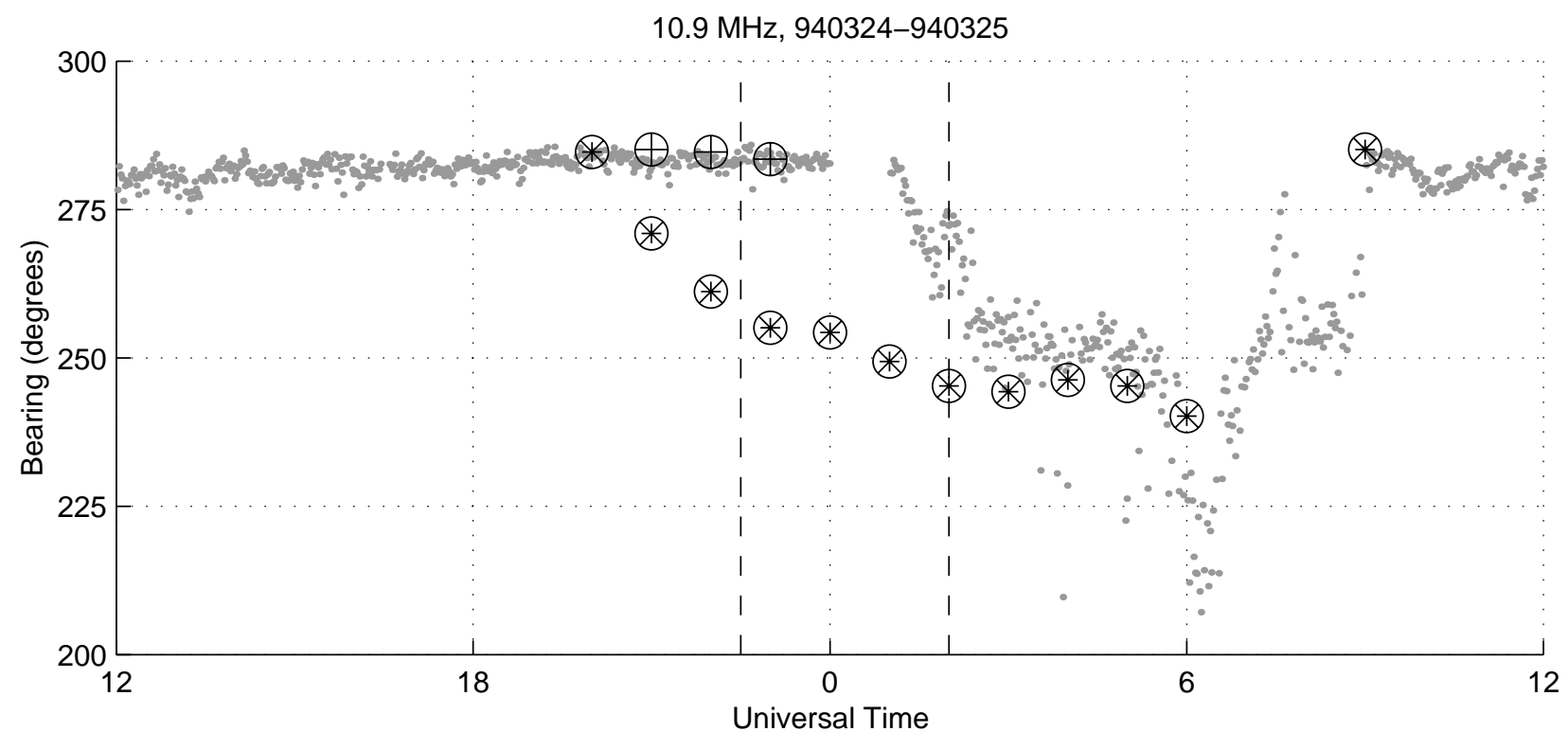

Figure 1. Observed and simulated bearings of a $10.945 \mathrm{MHz}$ signal as a function of time between 24 March 1994, 1200 UT and 25 March 1994, 1200 UT. Note that there is a data gap just after 0000 UT. The values obtained from the ray tracing simulation are given by the symbols $\otimes(\Delta=70 \%)$ and $\oplus(\Delta=40 \%)$. The vertical dashed lines indicate the earliest and latest times of trough opening for days of low geomagnetic activity at the end of March 1994.

The ray tracing simulation has been undertaken for an interval from $2000 \mathrm{UT}, 24$ March to $0900 \mathrm{UT}$, 25 March using a numerical ray tracing code [9]. In order to explain the off-GCP observations two potential mechanisms were investigated: a) two-hop propagation with the ground reflection via non-specular scatter [10] from the sea-surface at locations to the south of the trough, and b) refraction along the electron density gradients in the equatorward wall of the trough (the electron density poleward of the trough was too weak to support propagation). A background electron density model consisting of two Chapman layers, representing 
the $\mathrm{E}$ and $\mathrm{F}$ regions, with a linear gradient in electron density from the geographic equator has been adopted. The key parameters of the electron density model (critical frequency, critical height and scale height of each layer) were based on values obtained from the International Reference Ionosphere (IRI) [11] for the selected interval. The location of the trough was calculated according to the model of Halcrow and Nisbet [2]. However, in the ray tracing code a simplified version of this trough model was implemented in which it is assumed that the trough walls lie at constant values of geomagnetic latitude (for a given UT and $A_{p}$ ) and the trough ends are at constant geomagnetic longitude (again for a given UT and $A_{p}$ ). The position of the trough calculated for several times is illustrated in Figure 2. The maximum electron density reduction in the main trough, $\Delta$ is assumed to be either $70 \%$ or $40 \%$ below the ambient (i.e. troughless) value, while this perturbation decreases to zero over a few degrees of geomagnetic latitude once outside of the main trough. Close to the beginning of the interval chosen for the simulation (2100 UT) the trough covers about the last third of the GCP but the depleted electron density does not prevent propagation along on the great circle direction (see Figure 1). By 0000 UT, the trough covers the entire length of the GCP and so prevents GCP propagation since the maximum usable frequency (MUF) within the trough is reduced to below the signal frequency. At $0600 \mathrm{UT}$, although the trough closes just to the west of the receiver, the signals are still off great circle since the first ionospheric reflection of the $2 \mathrm{~F}$ mode remains within the trough. At $0900 \mathrm{UT}$, less than the first quarter of the GCP is covered by the trough and therefore the $2 \mathrm{~F}$ mode reflection points are outside of the trough and the signals are received along the great circle path.
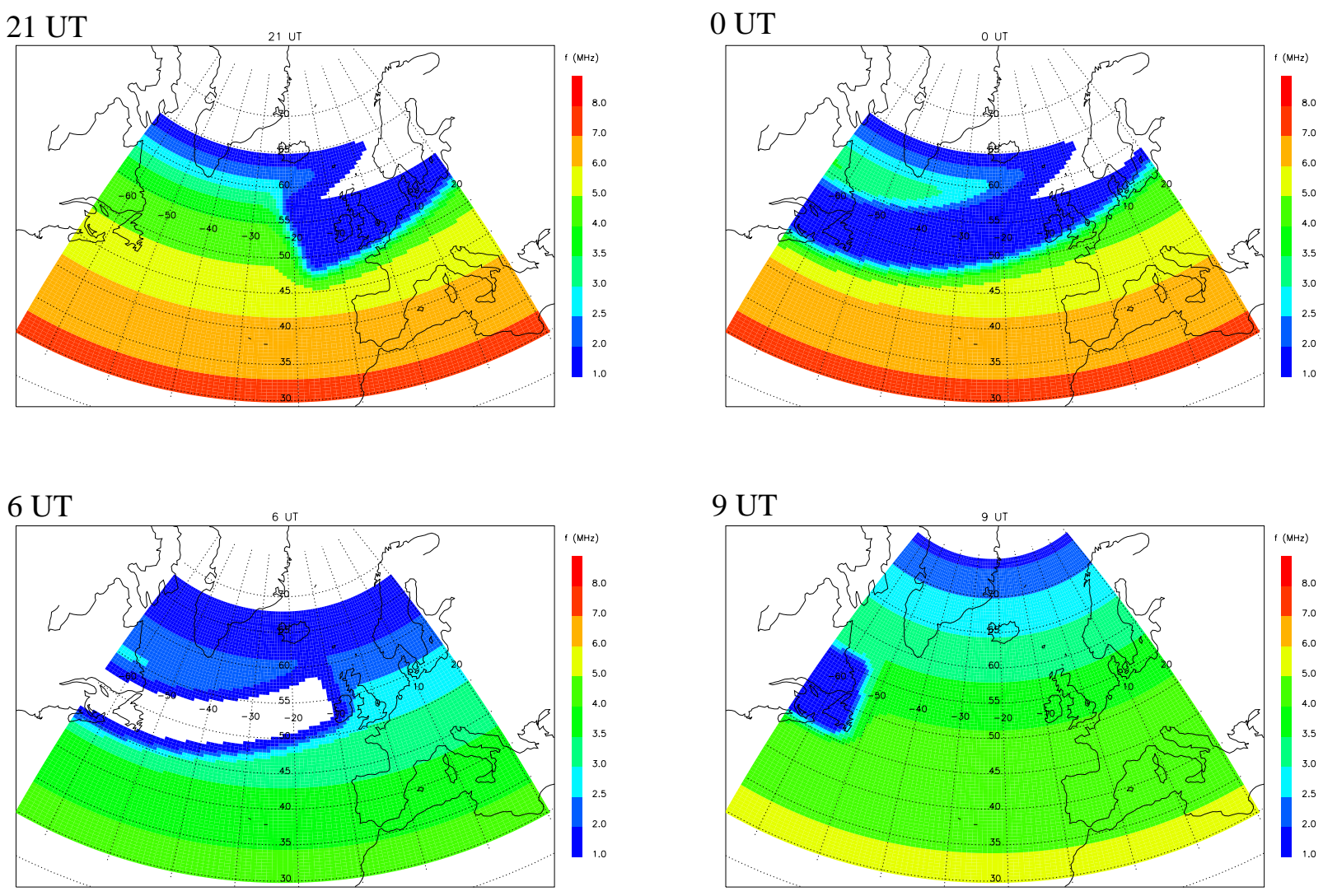

Figure 2. Maps of F-region critical frequency as a function of time. The location of the trough (70\% depletion) can clearly be seen.

As noted above, when the trough is present great circle propagation is not supported since the MUF within the trough is considerably reduced. However, there are directions in which propagation is supported to the south of the trough (e.g. the locations where rays are present at the earth's surface after both one and two hops are given in Figure 3). When calculating the second hop the ray tracing program usually assumes a specular reflection from the ground. However, this clearly does not result in any rays landing near to the receiver site (e.g. panels $b$ and $c$ of Figure 3). It is well known that since the sea is a rough surface then the radio wave will be to be scattered in many directions leading to so-called sidescatter [10]. An expression which relates the scattering coefficient, $\mathrm{R}_{\mathrm{sc}}$ (in $\mathrm{dB}$ ) as a function of the angle, $\alpha$ (in degrees) between the 
scattering wave and the ordinary reflected wave based on the experimental measurements of Miya and Kanaya [12] is given by:

$$
R_{s c}(\alpha)=-0.52 \alpha
$$

In order to take seascatter into account the ray tracing was conducted in two parts. Firstly rays were directed from the transmitter site until they returned to the ground or sea (i.e. the first hop, see Figure 3). Then each point where a ray landed was taken as the origin for a further set of rays, launched in all directions, each with a relative power given by Equation 1. The power and direction of arrival of rays in this second set which landed near the receiver were then recorded. The simulated direction of arrival of the seascattered rays has been plotted as a function of time together with the experimental observations in Figure 2 for two values of peak density perturbation caused by the trough $(\Delta)$. It should be noted that at times where only a datum for $\Delta=70 \%$ exists this implies that the result for $\Delta=40 \%$ is indistinguishable on the figure. With $\Delta=70 \%$, the simulated bearing turns southward from 2100 UT onward since there is insufficient ionisation within the trough to support GCP propagation at this frequency. The poor agreement with the experimental data continues until about 3 UT after which time the agreement between simulation and experiment is reasonable. If $\Delta=40 \%$, the deviation from the GCP occurs at $0000 \mathrm{UT}$, and the results are more consistent with observations although the effect of the trough is about an hour earlier in the simulation than in the experiment. However, it should be noted that the trough model employed [2] is based on the results of a statistical study and that there is some variation in the opening times of the trough under given conditions. Marked on Figure 2 (vertical dashed lines) are the earliest and latest times of opening of the trough inferred from the observed southward turning in bearing during the period 21 March 1994 to 28 March 1994 (a period during which the Ap values are roughly similar). Allowing the simulated opening time of the trough to be delayed by about an hour is therefore consistent with the uncertainty in the model and thereby the fit to the data could be further improved.
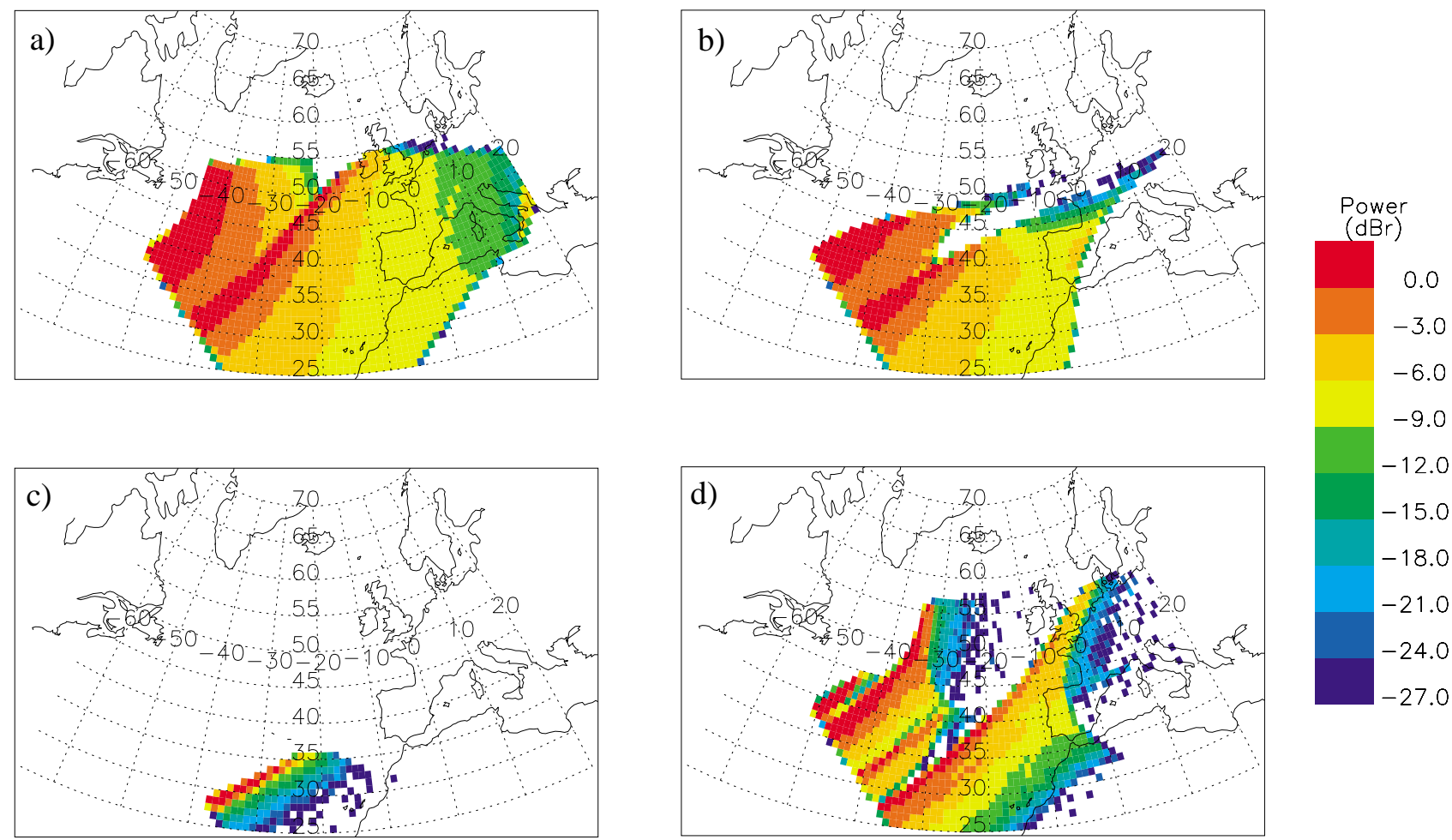

Figure 3. Map of relative power at ground level for the following conditions, a) 0000 UT with no trough present, $b$ ), c), and d) at 0000 UT, 0600 UT and 0900 UT respectively with trough present.

Rogers et al [8] suggested that the off-great circle propagation observed experimentally may have arisen because of reflections from the ionospheric gradients present on the equatorward wall of the trough. The viability of such a mode of propagation has been tested by ray tracing simulation. The simulated bearing offset from GCP is found to be strongly dependent on the location of the equatorward wall of the trough. The simulated bearing at times of 0000 UT and 0300 UT is presented in Figure 4. At 0000 UT, the azimuths 
are to the south of those observed (Figure 2), although this is likely to occur as a consequence of the uncertainty in the opening time of the trough. At 0300 UT, the bearings are slightly to the south of those calculated for 0000 UT but are still well to the north of the observed values.

Of the two potential mechanisms simulated above it would appear that the seascatter from regions well to the south of the trough produces results which are close to the observations provided that the reduction in electron density within the trough is lower than that given by the model of Halcrow and Nisbett [2] and that some variation in the trough opening time is allowed. It should be noted that this particular trough model is derived from topside measurements and there is little reason to believe that the electron density perturbation in the trough is height invariant. Furthermore, the ambient ionospheric properties have been derived from the IRI which is known to be less accurate at high latitudes. The simulations outlined here of propagation via the density gradients in the trough wall for the $4490 \mathrm{~km}$ path from Canada to the U.K. do not reproduce the large bearing deviations observed. It is, however, anticipated that the detailed nature of the propagation over shorter (i.e. 1-hop) trough affected paths will be much more dependent upon the gradients in the trough.

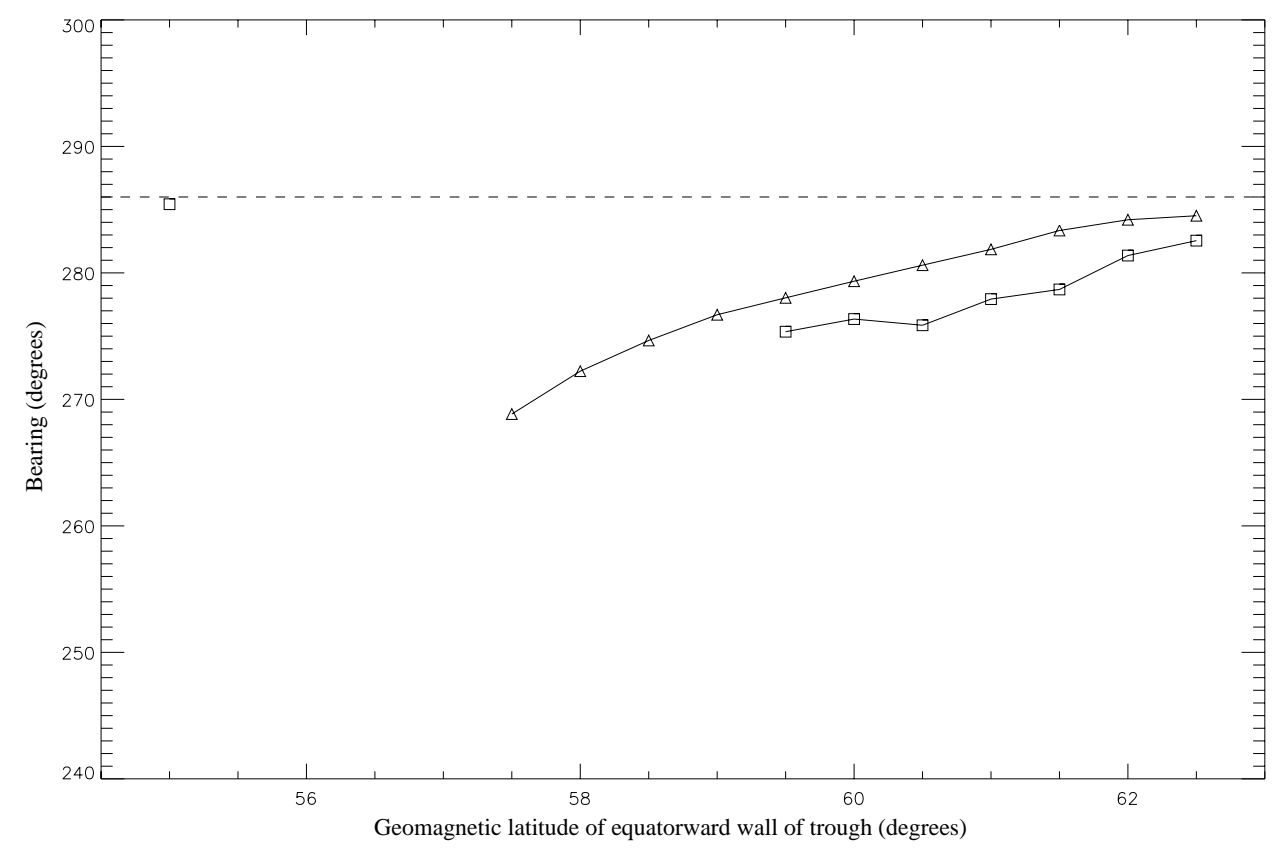

Figure 4. Simulated bearing of a $10.9 \mathrm{MHz}$ signal as a function of the position of the equatorward wall of the trough at a) 0000 UT (triangles) and b) 0300 UT (squares). The horizontal dashed line indicates the great circle direction.

\section{RECENT OBSERVATIONS, UPPSALA TO LEICESTER}

In order to obtain additional information about the trough over northern Europe with the aim of improving the ionospheric models for this region, a new experiment has been established (operational since October 2000) between Uppsala, Sweden and Leicester, U.K. The transmitter is located in Uppsala and radiates various modulations on frequencies according to a computer based schedule. The signals are received on a 6-channel superresolution direction finding system located $15 \mathrm{~km}$ south of Leicester, U.K. (see Figure 5). In the current schedule, a 1667 baud, 13-bit Baker coded signal of two-seconds duration is transmitted twice in a thirty second interval on one of six frequencies $(4.64,6.95,10.39,11.12,14.36$, and $18.38 \mathrm{MHz}$ ) which span the lower half of the HF band. The use of a Barker coded signal serves to increase the signal-to-noise ratio of the received signal through a despreading process and, importantly, acts as an aid to signal recognition. Since both transmitter and receiver system clocks are synchronised to GPS, the direction-of-arrival can be measured as a function of absolute time-of-flight.

As an additional diagnostic, a BR Communications chirpsounder system has also been deployed with the transmitter collocated with the main transmitter in Uppsala and the receiver at the University of Leicester. Oblique ionograms from the Uppsala transmissions are stored every 5 minutes except at 15 and 45 minutes past the hour when soundings taken over the Inskip to Leicester path are recorded. 


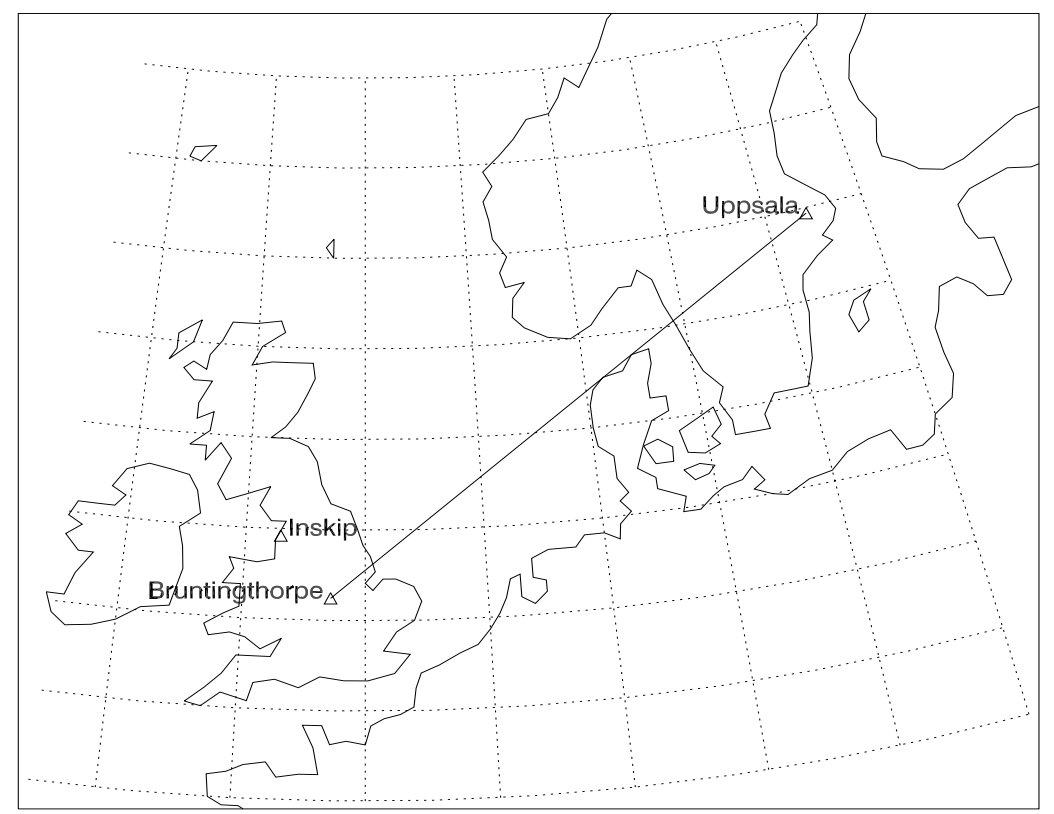

Figure 5. Map showing the location of the transmitter and receiver sites.

Some typical observations are presented in Figure 6. During the day (top-left panel) 1-hop, 2-hop and 3-hop F-region propagation is observed (this is confirmed by simultaneous oblique ionogram measurements which are not shown). The azimuthal angle of arrival (second-left) is close to the GCP $\left(46^{\circ}\right)$ for each mode while the elevation angle (third-left) is just under $20^{\circ}$. The frequency spectrum shows a narrow peak $(2 \mathrm{~Hz}$ at $-10 \mathrm{~dB}$ ). At night (top-right) two modes are present, a narrow one at a delay of just under $8 \mathrm{~ms}$ (i.e. at about the same delay as the 3F daytime mode) and a second mode which occurs at a longer delay and is more spread. The azimuthal direction of both modes is about $20^{\circ}$ to the north of the GCP although there is a linear variation in the bearing with time-of-flight. The elevation angles are again below $20^{\circ}$. The frequency spectrum is somewhat broader than the daytime case $(\sim 10 \mathrm{~Hz}$ at $-10 \mathrm{~dB})$, the reasons for which will be discussed below.

The bearing and time-of-flight (TOF) measurements made over three days (noon on 20 November (day number 324) to noon on 23 November 2001 (day number 327)) are shown in Figure 7. During the day, the signals at each frequency exhibit several modes with delays typically in the range 5 to $8 \mathrm{~ms}$, corresponding, in this case, to $1 \mathrm{~F}, 2 \mathrm{~F}$, and $3 \mathrm{~F}$ propagation, and bearings close to the great circle $\left(46^{\circ}\right)$. The two lowest frequencies form an exception to this since they are strongly attenuated during the day leading to either no detected signal (e.g. $4.64 \mathrm{MHz}$ at noon on day 325) or a weak signal which results in a poor measurement of the azimuth (e.g. $6.95 \mathrm{MHz}$ at noon on day 325). As expected, the daytime propagation begins later and ends earlier with increasing frequency. At night, a wider variety of effects in both azimuth and time-offlight are observed. On the first night (i.e. day 324-325) the two highest frequencies (14.36 and $18.38 \mathrm{MHz})$ do not propagate (this is also the case for day 325-326), 10.39 and $11.12 \mathrm{MHz}$ propagate via an E-region mode at bearings of about $40^{\circ}$ north of the GCP, $6.95 \mathrm{MHz}$ is via a weak F-region mode until just after midnight and thereafter on-great circle via an E-region mode, while $4.64 \mathrm{MHz}$ propagates on-great circle throughout the night. On the second night (day 325-326) just after midnight propagation occurs on a mode with a relatively long time-of-flight $(8-10 \mathrm{~ms}$ ) at frequencies 10.39 and $11.12 \mathrm{MHz}$ (and to a lesser extent $6.95 \mathrm{MHz}$ ), this mode is associated with bearings simultaneously to both the north and south of the GCP. The most striking behaviour can be observed on the third night (day 326-327) where, on most frequencies, a propagation mode appears at a long time-of-flight (up to $16 \mathrm{~ms}$ ) almost immediately after daytime propagation has finished (about $2030 \mathrm{UT}$ ). Over the next six hours the time-of-flight decreases reaching a steady value of about $7 \mathrm{~ms}$ by $0230 \mathrm{UT}$. From about 05 UT until the mode disappears at $0630 \mathrm{UT}$ (for $6.95 \mathrm{MHz}$, earlier for other frequencies) the time-of-flight increases by about $0.5 \mathrm{~ms}$. At times around midnight a second mode at longer delays $(\sim 11 \mathrm{~ms})$ is present at 10.39 and $11.12 \mathrm{MHz}$ (also see Figure 6). It is interesting to note that this night is one of only two in over a year of observations in which the signal at $18.38 \mathrm{MHz}$ exhibits this behaviour (albeit rather weakly). The bearing of this mode, which is strongly offgreat circle and mostly to the north of the GCP, displays both temporal and frequency dependence. In time, the bearing deviation from great circle decreases as the time-of-flight decreases (e.g. for $11.12 \mathrm{MHz}$, from about $40^{\circ}$ at $2030 \mathrm{UT}$ to $20^{\circ}$ at $0230 \mathrm{UT}$ ), while the bearing deviation from great-circle observed at 
$6.95 \mathrm{MHz}$ is smaller than at either 10.39 or $11.12 \mathrm{MHz}$. In Figure 8 the Doppler frequency and Doppler spread for the $11.12 \mathrm{MHz}$ signal are presented. As might be expected, the rapid decrease in time-of-flight is accompanied by a strong positive Doppler shift $\left(\sim 20 \mathrm{~Hz}\right.$, corresponding to a velocity $\left.\sim 500 \mathrm{~ms}^{-1}\right)$. As already discussed the Doppler spread is somewhat higher than that found during the day.

The feature observed on day 326-327, where a long time-of-flight is accompanied by bearing deviations and Doppler frequency offsets is a relatively common occurrence (see Table 1). In the winter and spring months, this phenomenon is observed on over $40 \%$ of nights, while in the summer and autumn it is less frequent. Examination of Table 1 also reveals that bearing deviations to the north of the GCP are 3-4 times more likely to occur than those to the south and that this is independent of season.

The likely cause of the effects observed is evident in the two ionograms from the morning of day 327 presented in Figure 9. In the top panel, the ionogram taken on the Uppsala-Leicester path exhibits a number of features. At a relative delay of about $2 \mathrm{~ms}$ and a critical frequency of just over $4 \mathrm{MHz}$ are returns from the normal ionospheric F-layer. However, above this, extending to a maximum frequency of about $14 \mathrm{MHz}$ is a more diffuse set of echoes. In the short-path ionogram (bottom panel) similar features can also be found although somewhat more pronounced. These diffuse returns which probably result from scattering from irregularities either in the poleward wall of the trough or in the auroral oval are clearly responsible for the bearing deviations, the enhanced time-of-flight and the increased Doppler spread. Although a detailed ray tracing simulation has yet to be performed, qualitatively it would seem that this explanation is consistent with all of the observations made to date.
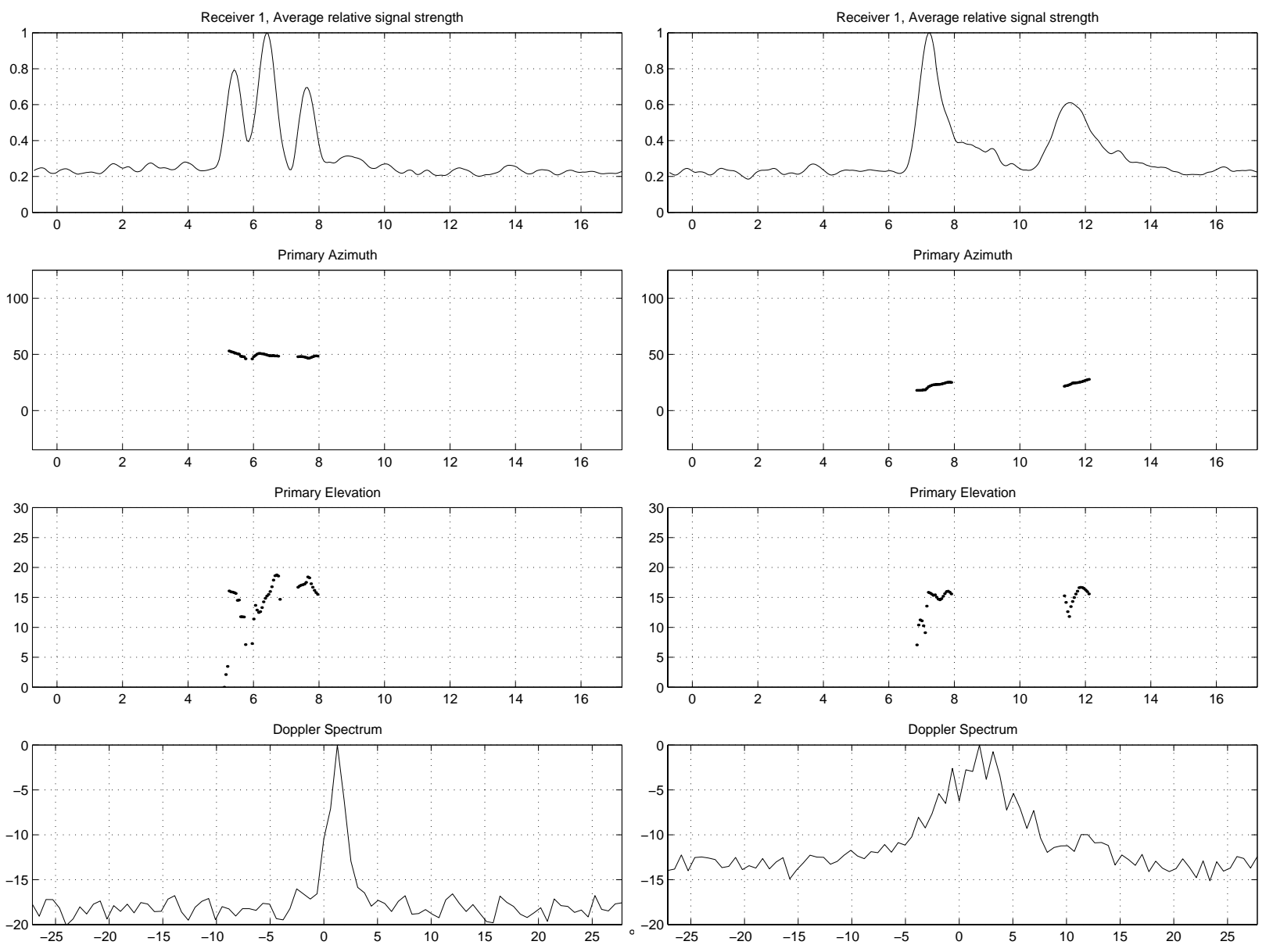

Figure 6. Example measurements made at $11.12 \mathrm{MHz}$ signals received at Bruntingthorpe on 23 November 2001 (day 327) for daytime (1134 UT, left) and night-time (0004 UT, right). From the top, the panels represent signal strength (linear, normalised) versus time-of-flight $(\mathrm{ms})$, azimuth $\left(^{\circ}\right)$ versus time-offlight, elevation $\left({ }^{\circ}\right)$ versus time-of-flight, and amplitude $(\mathrm{dB})$ versus Doppler frequency $(\mathrm{Hz})$. 

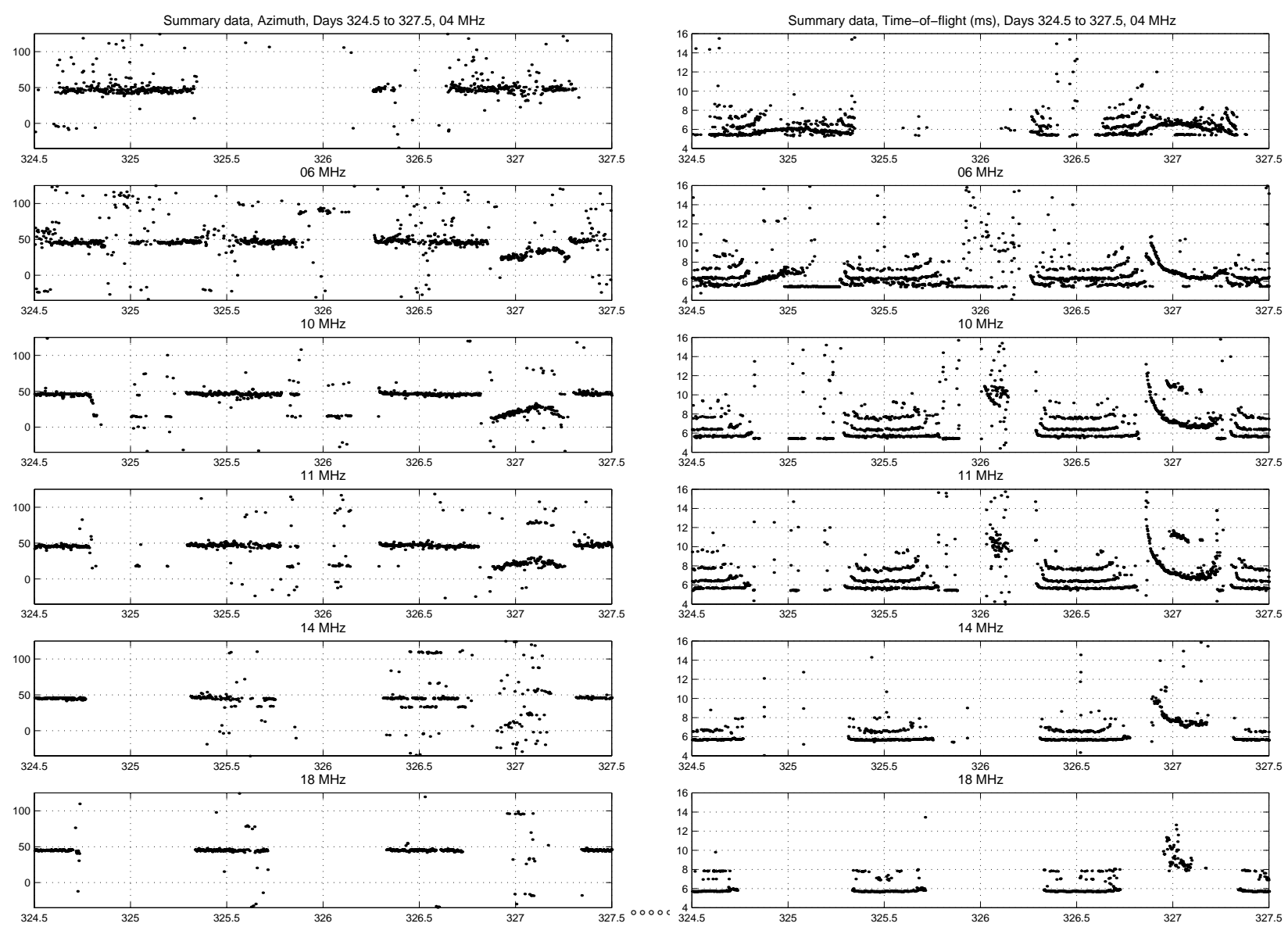

Figure 7. The azimuth $\left({ }^{\circ}\right.$, left) measured at the strongest mode and time-of-flight of each mode (ms, right) for 1200 UT, 20 November 2001 (day 324) to 1200 UT,23 November 2001 (day 327). The panels, from the top, represent frequencies of 4.64, 6.95, 10.39, 11.12, 14.36, and $18.38 \mathrm{MHz}$ respectively.
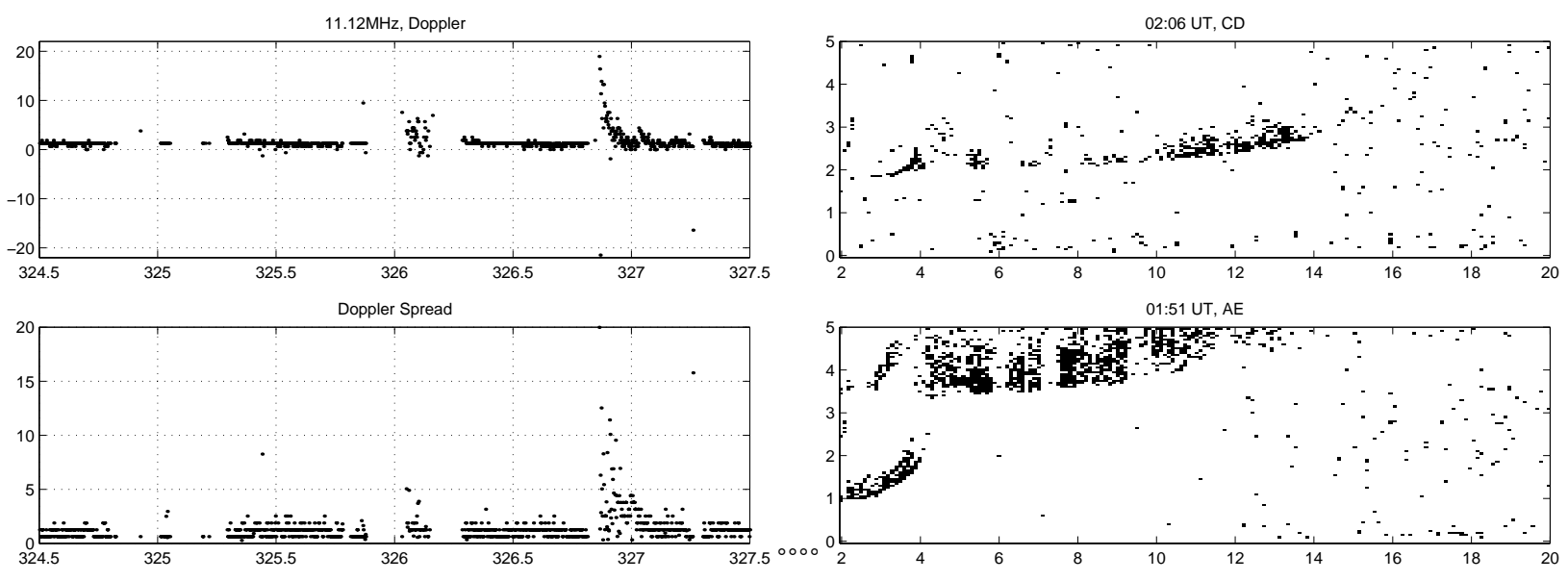

Figure 8. Doppler frequency and Doppler spread

Figure 9. Ionograms taken near to 0200 UT, 23 November 2001 (day 327). The top panel is over an oblique path from Uppsala, the bottom panel is for the short path. 
Table 1. Occurrence statistics of long time-of-flight propagation, and northward and southward bearing deviations (figures are \% of nights)

\begin{tabular}{cccc}
\hline Season & Long TOF & North & South \\
\hline Winter & 48 & 39 & 14 \\
Spring & 43 & 30 & 12 \\
Summer & 14 & 12 & 4 \\
Autumn & 26 & 23 & 8 \\
\hline
\end{tabular}

\section{CONCLUDING REMARKS}

A ray tracing simulation has been employed to investigate off great circle propagation on a $4490 \mathrm{~km}$ WestEast path in the presence of the mid-latitude trough. Two propagation mechanisms were studied, namely non-specular scatter from the sea at locations to the south of the trough and reflection from the walls of the trough. The results obtained for seascatter are, in general, in good agreement with the observations at a number of frequencies provided that the reduction in electron density caused by the trough is considered to be lower than that given by the Halcrow and Nisbett model [2] and that some (not unexpected) variation in the trough opening time is allowed. In contrast, ray tracing simulations of reflections from the equatorward wall of the trough did not reproduce the large bearing deviations observed over this long $(4490 \mathrm{~km}) \mathrm{path}$. It is, therefore, suggested that the dominant propagation mechanism resulting in the large deviations from the GCP over long paths along the trough is a combination of the suppression of the GC propagation resulting from the depleted electron density combined with multi-hop propagation supported by side scatter from the sea surface. It is, however, anticipated that for shorter paths the detailed directional characteristics of signals propagating over shorter trough affected paths will be heavily dependent upon the electron density gradients within the trough walls.

In order to obtain additional information about the trough over northern Europe, a new experiment has been established between Uppsala, Sweden and Leicester, U.K., the particular aim of which is the improvement and development of the ionospheric trough models for this region. Unlike the earlier experiments [8] in which the signal azimuth, amplitude and Doppler frequency characteristics were recorded, the new measurements include the elevation angle of arrival and since they are of a time synchronised pulsed transmission time-of-flight characteristics may be incorporated in the analysis.

Analysis of the measurements will initially be directed towards (a) experimentally determining the accuracy of the currently available models of the trough by a comparison of ray tracing simulations with the measurements, and (b) improvement of the accuracy of the models by imposing physically realistic refinements to give a better fit to the experimental data.

\section{REFERENCES}

1 Moffett, R.J. and Quegan, S. The mid-latitude trough in electron concentration of the ionospheric Flayer: a review of observations and modelling. Journal of Atmospheric and Terrestrial Physics, 1983, 45, pp 315-343.

2 Halcrow, B.W. and Nisbet, J.S. A model of the F2 peak electron densities in the main trough region of the ionosphere. Radio Science, 1977, 12, pp 815-820.

3 Rothkaehl H., Stanislawska I., Leitinger R., Tulunay Y. Application of a trough model for telecommunication purposes. Physics and Chemistry of the Earth Part C - Solar-Terrestrial and Planetary Science, 2000, 25, (4), pp 315-318.

4 Hanbaba B. COST 251: Improved Quality of Service in Ionospheric Telecommunication Systems Planning and Operation. Final Report. Space Research Centre, Warsaw, Poland, 1999.

5 Kersley L., Pryse S.E., Walker I.K., Heaton J.A.T., Mitchell C.N., Williams M.J., Willson C.A. Imaging of electron density troughs by tomographic techniques. Radio Science, 1997, 32, pp 16071621. 
6 Mitchell C.N., Kersley, L. and Pryse S.E. The effects of receiver location in two-station experimental ionospheric tomography. Journal of Atmospheric and Solar Terrestrial Physics, 1997, 59, pp 14111415.

7 Jones D.G., Walker, I.K. and Kersley, L. Structure of the poleward wall of the trough and the inclination of the geomagnetic field above the EISCAT radar. Annales Geophysicae, 1997, 15, pp 740746.

8 Rogers N.C., Warrington, E.M. and Jones, T.B. Large HF bearing errors for propagation paths tangential to the auroral oval. IEE Proceedings on Microwaves, Antennas and Propagation, 1997, 144, pp 91-96.

9 Jones R.M. and Stephenson, J.J. A Versatile Three-Dimensional Ray Tracing Computer Program for Radio Waves in the Ionosphere. UT Report 75-76, U.S. Department of Commerce, Washington D.C., October 1975.

10 CCIR. Ground and Ionospheric Side- and Back-scatter (Question 32/6). Report 726-2, XVIIth Plenary Assembly, Düsseldorf, Reports of the CCIR (also decisions), Annex to Vol VI, Propagation in Ionised Media, CCIR International Radio Consultative Committee, Geneva, 1990.

11 Bilitza, D. (ed.). International Reference Ionosphere 1990. NSSDC 90-22, Greenbelt, Maryland, USA, 1990.

12 Miya, K. and Kanaya, S. Radio propagation prediction considering scattering wave on the earth's surface. Report of Ionosphere Research in Japan, 1955, 9, (1), pp 1-15. 